\title{
Caracterización clínica y desenlaces en neonatos con catéter central venoso o arterial
}

\section{Clinical characterization and outcomes of neonates with venous and/or arterial central catheter}

Lina María Martínez-Sánchez, ${ }^{1}$ Lucelly López-López, ${ }^{2}$ Luis Felipe Álvarez-Hernández, ${ }^{3}$ Camilo Ruiz-Mejía, ${ }^{4}$ Laura

Isabel Jaramillo-Jaramillo, ${ }^{5}$ Mariana Roldán-Isaza, ${ }^{6}$ Juan Diego Villegas-Alzate ${ }^{7}$

\begin{abstract}
Resumen
ANTECEDENTES: La cateterización intravascular es un procedimiento para insertar un catéter en el espacio endoluminal, con el fin de administrar fluidos, fármacos o determinar constantes fisiológicas.

OBJETIVO: Identificar los factores asociados con la mortalidad en recién nacidos con un catéter central en tres instituciones de alto nivel de complejidad de Medellín, Colombia. MATERIALES Y MÉTODOS: Estudio observacional, analítico, de cohorte retrospectiva llevado a cabo en neonatos a quienes se colocó un catéter central venoso o arterial en la unidad de cuidados intensivos. Fuente de información: historias clínicas. Se estimaron las frecuencias absolutas y relativas para resumir las variables cualitativas y cuantitativas: se resumieron con mediana y rango intercuartílico. Para determinar qué variables se asociaban con la muerte se estimaron pruebas de $\chi^{2}$ y RR con IC95\%.

RESULTADOS: Se incluyeron 149 neonatos: 56 (37.6\%) de sexo femenino y 93 del masculino (62.4\%). La principal forma de parto fue vértice espontáneo para 78 (52.3\%) neonatos y $84(56.4 \%)$ prematuros. En cuanto a peso adecuado para la edad gestacional lo tuvieron $107(71.8 \%)$ y $32(21.5 \%)$ fallecieron durante la atención. La cromosomopatía y la hipertensión pulmonar se relacionaron con incremento en la mortalidad.

CONCLUSIÓN: Se lograron determinar las principales características clínicas de los pacientes a quienes se instaló un catéter intravascular, que concuerdan con lo descrito en la bibliografía y permiten tener mayor certeza, de manera anticipada, respecto de los neonatos que podrán requerir este tipo de intervenciones.

PALABRAS CLAVE: Catéter; fármacos; neonatos; arterial; Colombia; hipertensión pulmonar.
\end{abstract}

\section{Abstract}

BACKGROUND: Intravascular catheterization is a procedure through which a catheter is inserted into the endoluminal space in order to administer fluids, drugs or determine physiological constants.

OBJECTIVE: To identify mortality related factors in neonates subjected to venous and/ or arterial central catheter in high level complexity institutions in Medellin, Colombia. MATERIAL AND METHODS: Original study with retrospective cohort analytical and observational design, was conducted in neonates with central venous and/or arterial catheters in the intensive care unit. The clinical histories were used as a secondary information source, sample size was not calculated. Absolute and relative frequencies were used to summarize qualitative variables and the quantitative variables were summarized with median and interquartile range. To estimate which variables were associated to death, chi square and RR were calculated with a 95\% confidence interval.

RESULTS: A total of 149 neonates were included: 56 (37.6\%) were female and 93 (62.4\%) were male. The main mode of delivery was spontaneous vertex for $78(52.3 \%)$ neonates and $84(56.4 \%)$ were preterm. $107(71.8 \%)$ had adequate weight for gestational age

\author{
${ }^{1}$ http://orcid.org/0000-0002-9555- \\ 0843 \\ ${ }^{2}$ http://orcid.org/0000-0002-1534- \\ $520 \mathrm{x}$ \\ ${ }^{3}$ http://orcid.org/0000-0002-0580- \\ 6463 \\ ${ }^{4}$ http://orcid.org/0000-0002-8151- \\ 0787 \\ ${ }^{5}$ http://orcid.org/0000-0002-2123- \\ 0847 \\ ${ }^{6}$ http://orcid.org/0000-0001-6981- \\ 8802 \\ 7 http://orcid.org/0000-0002-7544- \\ 9801
}

Universidad Pontificia Bolivariana, Colombia.

Recibido: 10 de junio de 2020

Aceptado: 16 de febrero de 2021

\section{Correspondencia}

Lina María Martínez Sánchez

linam.martinez@upb.edu.co

Este artículo debe citarse como: Martínez-Sánchez LM, López-López L, Álvarez-Hernández LF, Ruiz-Mejía C, Jaramillo-Jaramillo LI, Roldán-Isaza M, Villegas-Alzate JD. Caracterización clínica y desenlaces en neonatos con catéter central venoso o arterial. Acta Pediatr Méx 2021; 42 (3): 93-101. 
and $32(21.5 \%)$ died during care. Chromosomopathy and pulmonary hypertension were associated with increased mortality.

CONCLUSION: We were able to determine the main clinical characteristics of patients with intravascular catheter in the local population, which is consistent with what has been described in the literature and allows us to have greater certainty in advance regarding the neonates who may require this type of intervention.

KEY WORDS: Drugs; Neonates; Arterial; Catheter; Colombia; Pulmonary hypertension.

\section{ANTECEDENTES}

La cateterización intravascular es un procedimiento para insertar un catéter en el espacio endoluminal con el propósito de administrar fluidos, fármacos o determinar constantes fisiológicas. ${ }^{1,2,3}$ Los accesos venosos son fundamentales en el tratamiento médico moderno. Se iniciaron en el decenio de 1960 y, desde entonces, no han dejado de estudiarse y desarrollarse diferentes técnicas y tipos susceptibles de aplicarse en neonatos. $^{3}$

Los catéteres centrales, que se introducen en grandes vasos arteriales o venosos, son de gran utilidad en recién nacidos gravemente enfermos, que requieren atención en unidades de cuidados intensivos neonatales. ${ }^{1,2,3} \mathrm{El}$ calibre de los vasos de los neonatos, sobre todo en su extremo distal, dificulta mantener la cateterización periférica por largo tiempo. ${ }^{3-6}$ Existen varios tipos de catéteres centrales y los accesos umbilicales son los más frecuentes en los primeros días de vida. ${ }^{1,3}$ Los neonatos internados en unidades de cuidados intensivos e intermedios requieren, al menos en una ocasión, la colocación de un catéter central en más del 90\% de los casos.?

Las ventajas de la cateterización central en los neonatos son claras porque permiten tener una vía intravascular a largo plazo, administrar nutrición parenteral total, vasoactivos, antibióticos, hemoderivados, entre otras diferentes utilidades que no proveen las vías periféricas. ${ }^{5,8,9}$ Lo anterior, sin dejar de tomar en cuenta que los accesos centrales se asocian con tasas significativas de morbilidad y mortalidad neonatal. Comparados con los catéteres que se aplican a pacientes adultos, los dispositivos neonatales son de menor diámetro, lo que predispone a complicaciones mecánicas, como la obstrucción y la trombosis; además, incrementan el riesgo de bacteriemias asociadas con el catéter. ${ }^{1,6}$ En el Cuadro $\mathbf{1}^{1,3,6}$ se resumen algunas de las complicaciones asociadas con la aplicación de catéteres centrales. Por esto, a pesar de que los catéteres centrales son imprescindibles en las unidades de cuidados intensivos, la decisión de aplicarlos debe ser individualizada y basarse en las necesidades del recién nacido. ${ }^{10}$

Cuadro 1. Complicaciones asociadas con la aplicación de de catéteres centrales ${ }^{1,3,6}$

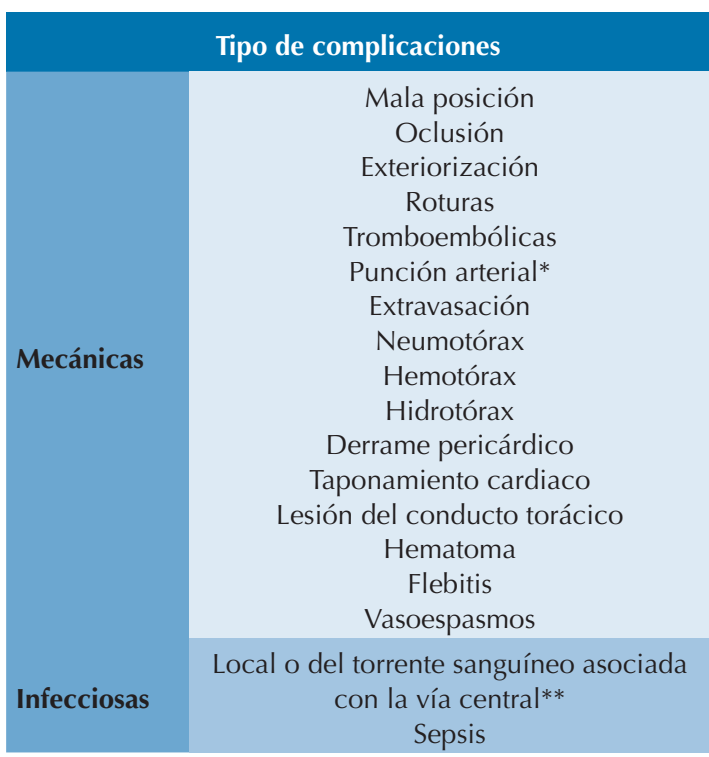

* Tratándose de un catéter venoso central

Las más comunes son las causadas por: Staphylococcus coagulasa negativa, Staphylococcus aureus, Enterococcus faecium y faecalis, Klebsiella pneumoniae, E. coli, Enterobacter cloacae y Pseudomonas aeruginosa 
Algunos autores reportan que la permanencia de los catéteres es muy variable, con un promedio de 13.5 días pero que puede llegar, incluso, hasta 98 días en los venosos centrales y percutáneos. Los principales motivos para retirar los catéteres venosos centrales son: finalización del tratamiento, pérdida accidental del acceso, sospecha de infección, infección diagnosticada, sepsis y muerte. ${ }^{3}$

Por lo anterior, el objetivo de este estudio fue: identificar los factores asociados con la mortalidad en recién nacidos con un catéter central en tres instituciones de alto nivel de complejidad de Medellín, Colombia.

\section{MATERIALES Y MÉTODOS}

Estudio observacional, analítico, de cohorte retrospectiva llevado a cabo en neonatos con un catéter central venoso o arterial atendidos en la unidad de cuidados intensivos de un hospital de Medellín, entre los meses de julio de 2016 y julio de 2018. Criterios de inclusión: neonatos (recién nacidos por parto o cesárea de 28 días o menos desde su nacimiento) que durante su estancia hospitalaria en la unidad de cuidados intensivos tuvieron un catéter central venoso o arterial. Se llevó a cabo un muestreo no probabilístico de casos consecutivos que cumplieran con los criterios de elegibilidad.

Fuente de información: historias clínicas. Unidad de análisis: neonatos con catéter central venoso o arterial, o ambos. De las historias clínicas se tomó la información de la estancia en cuidados intensivos y los diferentes desenlaces, entre ellos la mortalidad. Se diseñó un formulario para recolectar los datos; ahí se incluyeron las variables clínicas que permitieron responder a los objetivos del estudio. Para probar el instrumento de recolección y la base de datos se Ilevó a cabo una prueba piloto con 10 historias clínicas que permitió estandarizar la recolección, digitación y, de esta manera, tener un control de sesgos de información al momento de la ejecución. El control de sesgos de selección se efectuó mediante un estricto cumplimiento de los criterios de elegibilidad de la población, en cada una de las instituciones participantes.

La información recolectada se digitó en una base de datos de Excel, con restricción de valores de las variables para disminuir los posibles errores a la hora de digitar. Para verificar la calidad de la información obtenida se llevó a cabo una revisión periódica de los registros. Las variables cualitativas se resumieron con frecuencias absolutas y relativas y las cuantitativas con mediana y rango intercuartílico. Para establecer cuáles variables se asociaban con la mortalidad, se estimaron los riesgos relativos con sus intervalos de confianza y con regresión de Poisson con ajuste de errores estándar por la agrupación de hospital. El análisis se procesó en el programa SPSS versión 24. Por tratarse de una investigación retrospectiva los datos faltantes se reportan en los resultados. Se trató de variables que no afectaban el objetivo principal del estudio.

La investigación fue aprobada por el Comité de Ética de la Escuela de Ciencias de la Salud de la Universidad Pontificia Bolivariana; se consideró una investigación sin riesgo, según la resolución 008430 de 1993 del Ministerio de Salud de Colombia. La investigación contó con el aval de las tres instituciones donde fue llevado a cabo el estudio.

\section{RESULTADOS}

Se incluyeron 149 neonatos: $56(37.6 \%)$ de sexo femenino y 93 del masculino (62.4\%). La principal forma de parto fue vértice espontáneo para $78(52.3 \%)$ neonatos y 84 (56.4\%) prematuros. $107(71.8 \%)$ tuvieron peso adecuado para la edad gestacional y $32(21.5 \%)$ fallecieron durante la atención. El resto de los datos puede consultarse en el Cuadro 2. 
Cuadro 2. Características generales de los neonatos

\begin{tabular}{|c|c|c|}
\hline Característica & $\mathbf{n}$ & $\%$ \\
\hline \multicolumn{3}{|l|}{ Sexo } \\
\hline Femenino & 56 & 37.6 \\
\hline Masculino & 93 & 62.4 \\
\hline \multicolumn{3}{|l|}{ Lubchenco } \\
\hline $\begin{array}{l}\text { Pequeño para la edad } \\
\text { gestacional }\end{array}$ & 35 & 23.4 \\
\hline $\begin{array}{l}\text { Peso adecuado para la edad } \\
\text { gestacional }\end{array}$ & 107 & 71.8 \\
\hline $\begin{array}{l}\text { Grande para la edad } \\
\text { gestacional }\end{array}$ & 1 & 0.8 \\
\hline Sin datos & 6 & 4.0 \\
\hline \multicolumn{3}{|l|}{ Forma de parto } \\
\hline Vaginal espontáneo & 78 & 52.3 \\
\hline Vaginal inducido & 11 & 7.38 \\
\hline Cesárea & 55 & 36.9 \\
\hline Sin datos & 5 & 3.4 \\
\hline \multicolumn{3}{|l|}{ Prematurez } \\
\hline $\mathrm{Si}$ & 84 & 56.4 \\
\hline No & 63 & 42.3 \\
\hline Sin datos & 2 & 1.3 \\
\hline $\begin{array}{l}\text { Peso al nacer : mediana y } \\
\text { percentiles } 25 \text { y } 75\end{array}$ & \multicolumn{2}{|c|}{$2330(1550-2905)$} \\
\hline $\begin{array}{l}\text { Edad gestacional: mediana y } \\
\text { percentil } 25 \text { a } 75\end{array}$ & \multicolumn{2}{|c|}{$35.6(31.9-38.3)$} \\
\hline
\end{tabular}

Las alteraciones más frecuentes fueron: cardiopatías $(30.2 \% ; n=45)$ y pulmonares $(10.7 \% ; n=16)$.

Los principales antecedentes patológicos: síndrome de dificultad respiratoria $(42.3 \% ; n=63)$ y sepsis $(39.6 \% ; n=59)$. Los demás se mencionan en el Cuadro 3.

Otros antecedentes patológicos de menor frecuencia fueron: deshidratación $(1.3 \% ; n=2)$, policitemia $(1.3 \% ; \mathrm{n}=2)$ y síndrome de aspiración de líquido amniótico meconial $(2 \% ; n=3)$.

La principal ubicación del catéter fue la umbilical $(67.8 \% ; n=101)$ seguida de la epicutánea $(19.5 \% ; n=29)$; el $57.6 \%(n=86)$ tuvieron otras ubicaciones diferentes (subclavias, yugulares y femorales). Es importante tener en cuenta que no son excluyentes, los neonatos podían tener varios catéteres simultáneamente (umbilical y epicutáneo, por ejemplo).

Cuadro 3. Otros antecedentes patológicos de los neonatos

\begin{tabular}{|l|c|c|}
\hline Alteración* & $\mathbf{n}$ & $\%$ \\
\hline Dificultad respiratoria & 80 & 53.7 \\
\hline Sepsis & 59 & 39.6 \\
\hline Ictericia & 38 & 25.5 \\
\hline Anemia & 32 & 21.5 \\
\hline Asfixia perinatal & 30 & 20.1 \\
\hline Hipertensión pulmonar & 21 & 14.1 \\
\hline Ductus arterial persistente & 20 & 13.5 \\
\hline Hemorragia intraventricular & 16 & 10.7 \\
\hline Neumonía & 14 & 9.4 \\
\hline Displasia broncopulmonar & 10 & 6.7 \\
\hline Foramen oval permeable & 9 & 6.0 \\
\hline Enterocolitis necrosante & 8 & 5.4 \\
\hline Cromosomopatía & 8 & 5.4 \\
\hline Hipoglucemia & 5 & 3.4 \\
\hline Síndrome de abstinencia & 5 & 3.4 \\
\hline * No son excluyentes & & \\
\hline
\end{tabular}

Solo se encontró un caso de trombosis asociada con un catéter femoral derecho, en un recién nacido a las 31 semanas, pequeño para la edad gestacional (900 g) que tuvo sepsis temprana, síndrome de dificultad respiratoria, neumonía y anemia. Se le indicó tratamiento antitrombótico y fue dado de alta del hospital sin secuelas relacionadas con la prematurez. Cuadro 4

Cuadro 4. Desenlaces

\begin{tabular}{|l|c|c|}
\hline Desenlace & $\mathbf{n}$ & $\%$ \\
\hline Muerte & 32 & 21.5 \\
\hline Alta sin secuelas a corto plazo* & 73 & 49.0 \\
\hline Alta con secuelas a corto plazo* & 43 & 28.9 \\
\hline $\begin{array}{l}\text { Estancia hospitalaria que supera el } \\
\text { periodo neonatal }\end{array}$ & 34 & 22.8 \\
\hline
\end{tabular}

*Secuelas a corto plazo relacionadas con tromboembolismo 
Las principales morbilidades asociadas con la muerte intrahospitalaria, estadísticamente significativas, fueron: cromosomopatías (RR 4.07; IC95\%: 2.39-6.91), hipertensión pulmonar (RR 2.77; IC95\%: 1.54-4.99) y enfermedad pulmonar congénita (RR 2.33; IC95\%: 1.20-4.50), el resto se enlistan en el Cuadro 5. La asociación con la mortalidad se estimó con los riesgos relativos, intervalos de confianza, regresión de Poisson y ajuste de errores estándar por la agrupación de hospital.

Por lo que se refiere a la muerte no relacionada con trombosis se encontró que la cromosomopatía y la hipertensión pulmonar tuvieron mayor riesgo de muerte con RR 2.36 (IC95\%: 1.35-4.10) y 2.50 (IC95\%: 1.39-4.52), respectivamente.

\section{Cuadro 6}

Cuadro 5. Factores asociados con la mortalidad intrahospitalaria en recién nacidos con catéter

\begin{tabular}{|c|c|c|c|c|}
\hline Variables & & n $(\%)$ & RR (IC95\%) Crudo & p \\
\hline \multirow{3}{*}{ Sexo } & Femenino & $10-56(17.9)$ & 1 & 0.411 \\
\hline & Masculino & $22-93(23.7)$ & $1.32(0.68-2.59)$ & \\
\hline & $\begin{array}{l}\text { Pequeño para la edad } \\
\text { gestacional }\end{array}$ & 7-35 (20.0) & 1 & 0.765 \\
\hline \multirow[t]{4}{*}{ Lubchenco } & Peso adecuado & 24-107 (22.4) & $1.12(0.53-2.38)$ & \\
\hline & Grande & 0-1 (0.0) & Indeterminado & \\
\hline & Sin datos & $1-6(16.7)$ & NA & \\
\hline & Vaginal espontáneo & $20-78(25.6)$ & $1.28(0.67-2.46)$ & 0.44 \\
\hline \multirow[t]{2}{*}{ Forma del parto } & Vaginal inducido & $1-11(9.1)$ & $0.45(0,07-3.17)$ & \\
\hline & Cesárea & $11-55(20.0)$ & 1 & \\
\hline \multirow{2}{*}{ Prematurez } & Sí & $21-84(25.0)$ & $1.43(0.75-2.75)$ & 0.281 \\
\hline & No & $11-63(17.5)$ & 1 & \\
\hline \multirow{2}{*}{ Cardiopatía congénita } & Sí & $13-45$ (28.9) & $1.58(0.86-2.92)$ & 0.143 \\
\hline & No & $19-104(18.3)$ & 1 & \\
\hline \multirow{2}{*}{$\begin{array}{l}\text { Enfermedad pulmonar } \\
\text { congénita }\end{array}$} & Sí & 7-16 (43.8) & $2.33(1.20-4.50)$ & 0.012 \\
\hline & No & 25-133 (18.8) & 1 & \\
\hline \multirow{2}{*}{$\begin{array}{l}\text { Otras enfermedades } \\
\text { congénitas }\end{array}$} & Sí & $10-34(29.4)$ & $1.54(0.81-2.92)$ & 0.189 \\
\hline & No & 22-115 (19.1) & 1 & \\
\hline \multirow{2}{*}{ Sepsis } & Sí & $12-59(20.3)$ & $0.92(0.48-1.73)$ & 0.785 \\
\hline & No & $20-90(22.2)$ & 1 & \\
\hline \multirow{2}{*}{ Deshidratación } & Sí & $0-2(0.0)$ & Indeterminado & \\
\hline & No & $32-147$ (21.8) & 1 & \\
\hline \multirow{2}{*}{$\begin{array}{l}\text { Síndrome de insuficiencia } \\
\text { respiratoria }\end{array}$} & Sí & $16-63(25.4)$ & $1.37(0.74-2.52)$ & 0.319 \\
\hline & No & 16-86 (18.6) & 1 & \\
\hline \multirow{2}{*}{ Policitemia } & Sí & $1-2(50.0)$ & $2.37(0.57-9.82)$ & 0.234 \\
\hline & No & $31-147$ (21.1) & 1 & \\
\hline \multirow{2}{*}{$\begin{array}{l}\text { Síndrome de aspiración de líquido } \\
\text { amniótico meconial }\end{array}$} & Sí & $0-3(0.0)$ & Indeterminado & \\
\hline & No & $32-146$ (21.9) & 1 & \\
\hline \multirow{2}{*}{ Neumonía } & Sí & $3-14(21.4)$ & $0.997(0.35-2.86)$ & 0.996 \\
\hline & No & $29-135$ (21.5) & 1 & \\
\hline \multirow{2}{*}{ Diabetes materna } & Sí & $0-1(0.0)$ & Indeterminado & \\
\hline & No & $32-148(21.6)$ & 1 & \\
\hline
\end{tabular}




\begin{tabular}{|c|c|c|c|c|}
\hline \multirow{2}{*}{ Enterocolitis necrotizante } & Sí & $4-8(50.0)$ & $2.50(1.16-5.39)$ & 0.019 \\
\hline & No & $28-140(20.0)$ & 1 & \\
\hline \multirow{2}{*}{ Asfixia perinatal } & Sí & $9-30(30.0)$ & $1.55(0.80-2.99)$ & 0.191 \\
\hline & No & $23-119(19.3)$ & 1 & \\
\hline \multirow{2}{*}{ Cromosomopatía } & Sí & $6-8(75.0)$ & $4.07(2.39-6.91)$ & $<0.001$ \\
\hline & No & $26-141(18.4)$ & 1 & \\
\hline \multirow{2}{*}{ Ductus arterioso persistente (h) } & Sí & $9-20(45.0)$ & $2.50(1.36-4.61)$ & 0.003 \\
\hline & No & $23-128(18.0)$ & 1 & \\
\hline \multirow{2}{*}{ Ictericia } & Sí & 4-38 (10.5) & $0.42(0.16-1.11)$ & 0.081 \\
\hline & No & $28-111(25.2)$ & 1 & \\
\hline \multirow{2}{*}{ Enfermedad de membrana hialina } & Sí & 7-17 (41.2) & $2.17(1.11-4.24)$ & 0.023 \\
\hline & No & $25-132(18.9)$ & 1 & \\
\hline \multirow{2}{*}{ Anemia } & Sí & 5-32 (15.6) & $0.68(0.28-1.62)$ & 0.38 \\
\hline & No & $27-117(23.1)$ & 1 & \\
\hline \multirow{2}{*}{ Hipertensión pulmonar } & Sí & $10-21(47.6)$ & $2.77(1.54-4.99)$ & 0.001 \\
\hline & No & $22-128(17.2)$ & 1 & \\
\hline \multirow{2}{*}{ Hemorragia intraventricular } & Sí & $2-16(12.5)$ & $0.55(0.15-2.10)$ & 0.386 \\
\hline & No & $30-133(22.6)$ & 1 & \\
\hline \multirow{2}{*}{ Foramen oval permeable } & Sí & $2-9(22.2)$ & $1.03(0.29-3.64)$ & 0.964 \\
\hline & No & $30-139(21.6)$ & 1 & \\
\hline \multirow{2}{*}{ Hipoglucemia } & Sí & $0-5(0.0)$ & Indeterminado & \\
\hline & No & $32-144(22.2)$ & 1 & \\
\hline \multirow{2}{*}{ Displasia broncopulmonar } & Sí & $2-10(20.0)$ & $0.92(0.26-3.31)$ & 0.898 \\
\hline & No & $30-138(21.7)$ & 1 & \\
\hline \multirow{2}{*}{ Síndrome de abstinencia } & Sí & $0-5(0.0)$ & Indeterminado & \\
\hline & No & $32-143(22.4)$ & & \\
\hline
\end{tabular}

La asociación con la mortalidad se estimó con los riesgos relativos y sus intervalos de confianza, con regresión de Poisson y ajuste de errores estándar por la agrupación de hospital.

Cuadro 6. Factores asociados con mortalidad hospitalaria en niños con catéter*

\begin{tabular}{|l|c|ccc|}
\hline Variable & RR & \multicolumn{2}{|c|}{ IC95\% } & p \\
\hline Peso al nacer & 0.99 & 0.99 & 1.00 & 0.963 \\
\hline Edad gestacional & 0.89 & 0.76 & 1.04 & 0.153 \\
\hline $\begin{array}{l}\text { Cromosomopatía } \\
\text { Hipertensión pulmonar }\end{array}$ & 2.36 & 1.35 & 4.10 & 0.002 \\
\hline $\begin{array}{l}\text { Enfermedad pulmonar } \\
\text { congénita }\end{array}$ & 1.76 & 0.85 & 3.62 & 0.124 \\
\hline $\begin{array}{l}\text { Otras enfermedades } \\
\text { congénitas }\end{array}$ & 1.58 & 0.82 & 3.05 & 0.169 \\
\hline $\begin{array}{l}\text { Ictericia } \\
\text { Asfixia perinatal }\end{array}$ & 0.52 & 0.21 & 1.29 & 0.163 \\
\hline Análisis mutivariado & & 0.98 & 3.70 & 0.056 \\
\hline
\end{tabular}

*Análisis multivariado

\section{DISCUSIÓN}

En este estudio se encontró que más del 70\% de los recién nacidos tuvieron peso adecuado para la edad gestacional, el 56\% eran prematuros. El antecedente patológico más frecuente fue el síndrome de dificultad respiratoria (42\%), el $22 \%$ fallecieron y los principales factores de riesgo fueron: enfermedad pulmonar congénita, enterocolitis necrotizante, cromosomopatía, ductus arterioso, síndrome de dificultad respiratoria e hipertensión pulmonar.

En el contexto clínico, la hospitalización de neonatos en cuidados intensivos es común. En 
la bibliografía, en los diferentes estudios, pudo evidenciarse un comportamiento similar en cuanto a las características sociodemográficas poblacionales. Ters y colaboradores ${ }^{11}$ efectuaron un estudio con 2011 neonatos, 1370 de estos con catéter venoso central umbilical; las semanas promedio fueron $32+3$ días $( \pm 5.2)$, mientras que Cartwright y su grupo, ${ }^{12}$ en Australia, evaluaron las líneas centrales en neonatos entre los años 1984 y 2002. Encontraron 2186 cateterizaciones centrales, con promedio de 28.3 semanas de embarazo (mínimo 22.6, máximo 42.3 semanas). En los estudios mencionados se evidencia que, en promedio, los pacientes son prematuros, al igual que en esta investigación, donde estos tuvieron la mayor representación porcentual, así mismo en este trabajo como en el de Cartwright y colaboradores ${ }^{12}$ y El Ters y su grupo, ${ }^{11}$ aunque en algunas ocasiones los pacientes tuvieron peso adecuado para la edad gestacional en su mayoría fueron de bajo peso al nacer, característica acorde con la prematurez.

En este estudio hubo un predominio del sexo masculino; en los estudios reportados en la bibliografía, relacionados con este tema, la mayor parte no incluyen al sexo como variable; Hosseinpour y su grupo ${ }^{13}$ llevaron a cabo un estudio con 200 pacientes, la mitad con implantación percutánea y la otra mitad quirúrgica del catéter, de éstos 54 y 60 de 100 neonatos por grupo en total eran de sexo femenino, respectivamente. No se encontraron más estudios que incluyeran esta información por lo que no hay evidencia suficiente para establecer una relación entre esa variable y las otras planteadas en los estudios.

Las alteraciones congénitas no son infrecuentes en las unidades de cuidados intensivos y son enfermedades ampliamente estudiadas debido a las implicaciones que suponen para la atención médica del paciente. Dursun y colaboradores ${ }^{14}$ Ilevaron a cabo un estudio con 7450 recién nacidos internados en cuidados intensivos, de los que 1024 (13.7\%) tuvieron anormalidades con- génitas. El sistema más comúnmente afectado fue el cardiovascular en $68.8 \%$ de los pacientes, seguido del craneofacial y genitourinario con 23.8 y $17.8 \%$, respectivamente. Con menor porcentaje se encontraban el sistema nervioso, las extremidades, la piel, y el músculo esquelético, entre otros. La afección principal fue concordante con la encontrada en este estudio.

La población tratada en unidades de cuidados intensivos, sobre todo los recién nacidos con cateterización central, suele tener diversas comorbilidades, algunas de ellas graves. Enseguida se mencionan las principales comorbilidades asociadas con mayor riesgo de mortalidad intrahospitalaria identificadas en nuestro estudio que se contrastan con la información disponible al respecto.

La hipertensión pulmonar persistente del recién nacido es un padecimiento asociado con mayor morbilidad y mortalidad significativa en el periodo de transición entre la vida intra y extrauterina. Casi siempre es transitorio y secundario a otras causas: asfixia perinatal, aspiración de meconio o sepsis. ${ }^{15}$ En el estudio aquí reportado se identificó que la hipertensión pulmonar se asoció con mayor riesgo de mortalidad intrahospitalaria (RR de 2.77; IC95\%: 1.54-4.99). Un estudio Ilevado a cabo por Schindler y coautores ${ }^{16}$ en recién nacidos muy prematuros (menos de 32 semanas de gestación) entre 2007 y 2011 llegó a desenlaces similares: identificaron a la hipertensión pulmonar como un factor de riesgo para mortalidad neonatal (OR: 6.08; IC95\%: 4.41-8.38) en análisis univariado porque en el multivariado se encontró un OR de 3.06; IC95\%: 2.02-4.64) que comparándolo con el resultado de este estudio (OR: 2.50; IC95\%: 1.39-4.52) se evidencia que ambos suponen un riesgo de mortalidad significativo.

De igual forma, en el estudio de Schindler y su grupo $^{16}$ se reportó asociación entre la enfermedad de membrana hialina tratada con surfactante 
y mayor riesgo de mortalidad (OR univariado: 4.23; IC95\%: 3.12-5.73), hallazgo similar al encontrado en nuestro estudio (RR 2.17; IC95\%: 1.11-4.24); no obstante, en el ajuste multivariado no se encontró significación (RR 0.84; IC95\%: $0.55-1.27$ ), este mismo comportamiento se evidencia en el estudio aquí comunicado.

Las malformaciones congénitas son causas importantes de morbilidad y mortalidad neonatal en las unidades de cuidados intensivos. En nuestro estudio, dos malformaciones congénitas se asociaron con mayor mortalidad: la enfermedad pulmonar congénita (RR: 2.33; IC95\%: 1.204.50) y el ductus arterioso persistente (RR: 2.50; IC95\%: 1.36-4.61). Este último puede estar mayormente relacionado con la prematurez debido a que el $70 \%$ (14 de 20) de los recién nacidos con ductus arterioso persistente eran prematuros. Un estudio emprendido por Berry y colaboradores ${ }^{17}$ encontró hallazgos similares; identificaron a las malformaciones congénitas como un factor predictor de mortalidad y estancia hospitalaria prolongada (OR para mortalidad: 7.28; IC95\%: 3.69-14.36).

Los desenlaces de este estudio son semejantes a los de la bibliografía; sin embargo, entre las limitaciones destacan el pequeño tamaño de la muestra y la ausencia de estudios similares que identificaran factores asociados con mayor mortalidad en neonatos con cateterización intravascular.

\section{CONCLUSIÓN}

En este estudio se lograron determinar las principales características clínicas de los neonatos a quienes se instala un catéter intravascular y se llegó a desenlaces similares a los reportados en la bibliografía. Además, se identificaron los factores asociados con mayor mortalidad en la muestra de estudio, pero no se identificaron los estudios que evaluaran los factores asociados con mayor mortalidad en neonatos a quienes se coloca una un catéter intravascular; la mayor parte de los estudios no discrimina esta variable, a pesar de esto los desenlaces fueron similares a los reportados hasta la fecha.

\section{Agradecimiento}

A los pacientes y a las instituciones que que hicieron posible cada etapa de esta investigación.

\section{REFERENCIAS}

1. Soares BN, Pissarra $S$, Rouxinol-Dias AL, Costa $S$, Guimarães $\mathrm{H}$. Complications of central lines in neonates admitted to a level III Neonatal Intensive Care Unit. J Matern Fetal Neonatal Med 2018; 31 (20): 2770-6. doi: 10.1080/14767058.2017.1355902.

2. Imigo F, Elgueta A, Castillo E, Celedón E, Fonfach C, Lavanderos J, et al. Accesos venosos centrales. Cuad Cir 2011; 25: 52-8.

3. García HJ, Torres-Yáñez HL. Duración y frecuencia de complicaciones de los catéteres venosos centrales en recién nacidos. Rev Med Inst Mex Seguro Soc 2015; 53 Supl 3: S300-9.

4. Athanasio D, Marques De Lazzari LS, Pedrolo E, Mingorance P, Queiroz T, Reichembach MT. Peripherally inserted central catheter care in neonates: an integrative literature review. Rev Esc Enferm USP 2012; 46 (6): 1502-10.

5. Sellitto M, Messina F. Central venous catheterization and thrombosis in newborns: update on diagnosis and management. J. Matern Fetal Neonatal Med 2012; 25 (S4): 26-28.

6. Miguelena D, Pardo R, Morón-Duarte L. Complicaciones relacionadas con catéteres venosos centrales en niños críticamente enfermos. Rev Salud Pública 2013; 15 (6): 916-28.

7. Carrera S, Félix I, Guido O, Fernández L, Cordero G, Yllescas $E$, et al. Complicaciones de los accesos vasculares centrales en los recién nacidos del Instituto Nacional de Perinatología. Perinatol Reprod Hum 2016; 30 (4): 167-71.

8. Lambert I, Tarima S, Uhing M, Cohen SS. Risk factors linked to central catheter-associated thrombosis in critically ill infants in the neonatal intensive care unit. Am J Perinatol 2019; 36 (3): 291-95. doi:10.1055/s-0038-1667377.

9. Chen IL, Ou-Yang MC, Chen FS, Chung MY, Chen CC, Liu YC, et al. The equations of the inserted length of percutaneous central venous catheters on neonates in NICU. Pediatr Neonatol 2019; 60 (3): 305-10. doi:10.1016/j.pedneo.2018.07.011.

10. Castro F. Beneficios del catéter epicutáneo en el recién nacido. Rev Cubana Enfermer 2004; 20 (2): 1.

11. El Ters N, Eldridge W, Rogowski L, Claassen C, Lancaster T, Barnette $A$, et al. Central versus low-lying umbilical 
Martínez-Sánchez LN, et al. Catéter central en neonatos

venous catheters: A multicenter study of practices and complications. Am J Perinatol 2019; 36 (11): 1198-1204. doi: 10.1055/s-0038-1676482.

12. Cartwright DW. Central venous lines in neonates: a study of 2186 catheters. Arch Dis Child Fetal Neonatal Ed 2004; 89: F504-08. doi: 10.1136/adc.2004.049189.

13. Hosseinpour M, Reza M, Behdad S, Azarbad Z. Central venous catheterization in neonates: Comparison of complications with percutaneous and open surgical methods. J Indian Assoc Pediatr Surg 2011; 16 (3): 99-101. doi: 10.4103/0971-9261.83487.

14. Dursun A, Zenciroglu A, Hakan N, Karadag N, Saygili B, Aydin $B$, et al. Distribution of congenital anomalies in a neonatal intensive care unit in Turkey. J Matern Fetal Neonatal Med 2014; 27 (10): 1069-74. doi: 10.3109/14767058.2013.847420.
15. Jain A, McNamara PJ. Persistent pulmonary hypertension of the newborn: Advances in diagnosis and treatment. Semin Fetal Neonatal Med 2015; 20 (4): 262-71. doi: 10.1016/j. siny.2015.03.001.

16. Schindler T, Koller-Smith L, Lui K, Bajuk B, Bolisetty S; New South Wales and Australian Capital Territory Neonatal Intensive Care Units' Data Collection. Causes of death in very preterm infants cared for in neonatal intensive care units: a population-based retrospective cohort study. BMC Pediatr 2017; 21; 17(1): 59. doi: 10.1186/s12887-017-0810-3.

17. Berry MA, Shah PS, Brouillette RT, Hellmann J. Predictors of mortality and length of stay for neonates admitted to children's hospital neonatal intensive care units. J Perinatol 2008; 28 (4): 297-302. https://doi.org/10.1038sj. jp.7211904. 